\title{
The Combination of Octreotide and Midodrine is not Superior to Albumin in Preventing Recurrence of Ascites after Large-Volume Paracentesis
}

\author{
Khurram Bari ${ }^{1,2}$, Cecilia Minano ${ }^{1,2}$, Martha Shea ${ }^{2}$, Irteza B. Inayat ${ }^{1,2}$, Hashem J. Hashem ${ }^{1,2}$, \\ HoChong Gilles $^{3}$, Douglas Heuman ${ }^{3}$, and Guadalupe Garcia-Tsao ${ }^{1,2}$ \\ ${ }^{1}$ Digestive Diseases, Yale University School of Medicine, New Haven, CT, United States \\ ${ }^{2}$ Digestive Diseases, VA-CT Healthcare System, West Haven, CT, United States \\ ${ }^{3} \mathrm{GI} /$ Hepatology, Hunter Holmes McGuire VA Medical Center, Richmond, VA, United States
}

\section{Abstract}

\begin{abstract}
Background \& Aims-Large-volume paracentesis (LVP) is the treatment of choice for patients with cirrhosis and refractory ascites. However, LVP can lead to post-paracentesis circulatory dysfunction (PCD), which is associated with faster ascites recurrence and renal failure. PCD results from vasodilatation, which reduces effective blood volume, and is prevented by intravenous administration of albumin. Vasoconstrictors could be used instead of albumin and, with longer use, prevent PCD and delay ascites recurrence.
\end{abstract}

\begin{abstract}
Methods-We performed a multicenter, randomized, double-blind, placebo controlled trial to compare albumin with the vasoconstrictor combination of octreotide and midodrine in patients with refractory ascites who underwent LVP. Patients in the albumin group received a single intravenous dose of albumin at the time of LVP plus placebos for midodrine and octreotide $(n=13)$. Patients in the vasoconstrictor group received saline solution (as a placebo for albumin),
\end{abstract}

(c) 2012 The American Gastroenterological Association. Published by Elsevier Inc. All rights reserved.

Correspondence: Guadalupe Garcia-Tsao M.D., Section of Digestive Diseases, Yale University School of Medicine, New Haven, CT and VA-Connecticut Healthcare System, West Haven CT., 950 Campbell Ave, Digestive Diseases, 111-H, West Haven, CT 06516, Phone: 203-932-5711 Ext: 2206, 4696, Fax: 203-937-3873, guadalupe.garcia-tsao@yale.edu.

Publisher's Disclaimer: This is a PDF file of an unedited manuscript that has been accepted for publication. As a service to our customers we are providing this early version of the manuscript. The manuscript will undergo copyediting, typesetting, and review of the resulting proof before it is published in its final citable form. Please note that during the production process errors may be discovered which could affect the content, and all legal disclaimers that apply to the journal pertain.

Disclosures: Douglas Heuman has received research grants from Novartis (manufacturer of Octreotide LAR) and is a sub investigator on a study sponsored by Ikaria (manufacturer of Terlipressin).

Khurram Bari: No conflicts of interest exist

Cecilia Minano: No conflicts of interest exist

Irteza Inayat: No conflict of interest exist

Martha Shea: No conflict of interest exist

Hashem J Hashem: No conflict of interest exist

HoChong Gilles: No conflict of interest exist

Guadalupe Garcia-Tsao: No conflict of interest exist

Writing Assistance: None

Author contributions: Cecilia Minano, Irteza Inayat, Martha Shea and Hochong Gilles were involved in data acquisition, administrative and technical support. Hashem Hashem was involved in initial protocol writing, development of data collection forms and administrative support for local IRB approval. Khurram Bari was involved in acquisition of data, analysis and interpretation of data, statistical analysis and drafting the manuscript. Douglas Heuman was involved in administrative, technical support and study supervision. Guadalupe Garcia-Tsao was involved in study concept and design, obtaining funding, administrative support, study supervision and critical revision of the manuscript for important intellectual content. 
$10 \mathrm{mg}$ of oral midodrine (3 times daily), and a monthly, $20 \mathrm{mcg}$ intra-muscular injection of longacting octreotide $(n=12)$. Patients were followed until recurrence of ascites.

Results-The median times to recurrence of ascites were 10 days in the albumin group and 8 days in the vasoconstrictor group $(P=.318)$. There were no significant differences in PCD between the albumin group $(18 \%)$ and the vasoconstrictor group $(25 \%, P=.574)$. When ascites recurred, serum levels of creatinine were higher in the vasoconstrictor group $(1.2 \mathrm{vs} 0.9 \mathrm{mg} / \mathrm{dL}$ in the albumin group, $P=.051)$.

Conclusions-The combination of midodrine and octreotide after LVP is not superior to albumin in delaying recurrence of ascites or preventing PCD in patients with cirrhosis. Outcomes appear to be worse in patients given octreotide and midodrine.

\section{Keywords}

cirrhosis; circulatory dysfunction; renal failure; randomized clinical trial

Refractory ascites is a relatively common complication of advanced cirrhosis characterized by ascites unresponsive to maximal doses of diuretic therapy or ascites that cannot be removed because maximal dose of diuretics cannot be reached because of the development of diuretic-induced complications [1] . Currently, the first-line recommended therapy for refractory ascites is large volume paracentesis (LVP) plus intravenous albumin [2] Intravenous albumin is administered to prevent the development of the so-called "postparacentesis circulatory dysfunction (PCD)" which is defined as a significant increase ( $250 \%$ ) in plasma renin activity (PRA) 6 days after LVP. PCD results from a decreased effective arterial blood volume and is associated with faster recurrence of ascites, development of renal dysfunction and a higher mortality [3;4]. Albumin is used as a volume expander and is associated with the lowest rate of PCD when compared to other plasma expanders [3;5].However, albumin is expensive and at times its availability is limited. Additionally, the cause of decreased arterial blood volume appears to be worsening vasodilatation [4] and therefore another alternative to prevent PCD would be the use of vasoconstrictors. Several small randomized studies have in fact demonstrated that the rate of PCD is similar when comparing terlipressin (a potent vasoconstrictor) to albumin [6-8]. In these studies, terlipressin has been administered for short periods after LVP, similar to intravenous albumin. We hypothesized that longer administration of safer vasoconstrictors, such as the combination of octreotide plus midodrine, could not only be as effective as single dose albumin in preventing PCD but would also lengthen the time to recurrence of ascites.

The aim of this double-blind, double-placebo-controlled study, performed in patients with cirrhosis and refractory ascites, was to compare the effect of long-term administration of the combination of vasoconstrictors, octreotide/midodrine, vs. standard single dose albumin after LVP in the in the recurrence of ascites and the development of PCD.

\section{METHODS}

\section{Study Design}

The study is a prospective, randomized, double-blind double-placebo controlled trial comparing intravenous albumin to vasoconstrictors (combination of octreotide-LAR and midodrine) after large volume paracentesis (LVP) in patients with cirrhosis and refractory ascites. The study was started as a two-center (West Haven VA Medical Center, West Haven, CT; and Yale University, New Haven, CT) trial in Oct 2003 but due to slow patient enrollment, another center (Hunter Holmes McGuire VA Medical Center, Richmond,VA) 
was added in Sep 2008. The study was approved by the local institutional review boards (IRB).

\section{Study Patients}

Patients included in the study met the following criteria: age between 18 and 80 years, cirrhosis of any etiology diagnosed by liver biopsy or based on firm clinical grounds, refractory ascites per International Ascites Club definition, i.e. ascites that has failed to respond to standard treatment of dietary salt restriction and diuretics (spironolactone $400 \mathrm{mg}$ and furosemide $160 \mathrm{mg} /$ day) or appearance of diuretic-induced complications [9] . Patients with hepatic hydrothorax, small amount of ascites, recent (within 1 month) GI hemorrhage, active bacterial infection, cardiac failure, findings suggestive of organic renal disease, hepatocellular carcinoma, and baseline serum creatinine greater than 3.0 were excluded.

\section{Study Procedures}

After establishing eligibility and having signed informed consent, patients were placed on a low sodium diet and diuretics were held for a minimum of 3 days. On the day of index LVP, patients were weighed and their abdominal girth was measured. Baseline heart rate, systolic and diastolic blood pressure were measured. In addition to routine labs, blood samples for aldosterone and plasma renin activity (PRA) were obtained after patients had been supine for at least 20 minutes. LVP was performed following a standard method [10]. A sample of fluid was sent for cell counts and bacterilogical culture. Patients were then randomized to two groups: a) The control group received IV albumin at a dose of $8 \mathrm{~g} / \mathrm{L}$ of ascites removed plus intramuscular (IM) administration of $5 \mathrm{cc}$ of saline solution (octreotide placebo) to be repeated every month plus a tablet (midodrine placebo) to be administered three times a day; b) the study group received an IV infusion of saline solution (albumin placebo) plus octreotide LAR $20 \mathrm{mg} \mathrm{IM}$, to be repeated every month plus a tablet of $10 \mathrm{mg}$ of midodrine to be administered at a dose of $10 \mathrm{mg}$ orally three times a day. Albumin (or albumin placebo) infusion was initiated towards the end of index LVP, first dose of octreotide and midodrine (or octreotide and midodrine placebos) were administered immediately after completing index LVP. In order to ensure that investigators performing paracentesis were blinded to albumin (a yellow solution) vs. its placebo (saline, a colorless solution) the whole IV setup was covered with a brown paper bag and sleeve. Midodrine tablets were identical in appearance to placebo tablets. Total duration of index visit, including study procedures, was about 3 hours. Randomization was performed using a random permuted block design in 1:1 ratio and was stratified to allow equal enrollment of patients based on presence of baseline renal dysfunction defined as blood urea nitrogen $(\mathrm{BUN})>30$ or serum creatinine $>$ 1.5. The random treatment allocation codes were generated at an independent bio statistical center by the study statistician using SAS version 8.2. A list with allocation codes was sent to the pharmacy which assigned the participants to interventions based on allocation codes. Follow up visits were scheduled at day 6 and day 15 and then monthly after randomization. At each of follow up visit, detailed history and physical examination including any alcohol use, weight, abdominal girth, mean arterial pressure (MAP), routine labs, spot urine sodium and creatinine and blood samples for aldosterone and PRA were collected. Diuretics were restarted at day 6 after index LVP.

\section{Study Endpoints}

The primary end point was time to recurrence of ascites defined as the requirement of a repeat LVP, as indicated by the presence of moderate to severe ascites AND weight gain to 90-100\% of baseline weight AND increase in abdominal girth to $90-100 \%$ of baseline abdominal girth. Secondary end points were a) the development of PCD, defined as an increase in PRA by $>50 \%$ from baseline to a level $>4 \mathrm{ng} / \mathrm{mL} / \mathrm{h}$ at post-paracentesis day 6 [3], b) average changes in mean arterial pressure and heart rate from baseline as surrogates 
of the hyperdynamic circulatory state and c) development of hepatorenal syndrome defined as the development of de novo HRS or progression from type 2 to type 1 HRS. Criteria for diagnosis of hepatorenal syndrome were based on recommendations of International Ascites Club [11].

The study was to be terminated and the study medications discontinued under the following circumstances: development of primary end-point (i.e recurrence of ascites requiring LVP), death, liver transplant or completion of 6 months from randomization.

\section{Statistical Analysis}

Sample size was calculated based on the time to recurrence of ascites. The hypothesis was that recurrence of ascites would be longer in the study group (long-term administration of octreotide plus midodrine) compared to the control group (single dose intravenous albumin). In a trial that included a similar population of patients with cirrhotic ascites and that recruited patients from the Yale/West Haven VA centers, the median time to recurrence of ascites was 20 days [12]. According to our hypothesis, we estimated that the median time to recurrence of ascites would be 38 days in the study group and 20 days in the control group in a fixed duration of 6 months. This sample size estimate was calculated to detect the effect of treatment using logrank test of equality of survival curves assuming 5\% type-I error (2 sided) and an $80 \%$ power. According to an initial survey in hospitalized patients at this center, we assumed that the sample could be recruited in four years. However, recruiting patients from this patient population with advanced liver disease proved to be quite challenging and despite the recruitment of an additional study center we were unable to recruit the desired number of patients. Based on randomized trials using vasoconstrictors in the prevention of PCD [6;13] and a study that showed that midodrine leads to a significant improvement in effective arterial blood volume, each of which had sample sizes of 24-25 patients, , we decided on a sample size of $\sim 30$ patients. This would allow us to consider this as a proof-of-concept study that could provide evidence for the planning of large scale, multicenter trials.

Time to recurrence of ascites was calculated and event free survival was estimated in each group using Kaplan-Meier method with $95 \%$ confidence interval. Analysis for secondary outcomes included changes in plasma renin activity, pulse, MAP at day 6 from baseline and development of hepatorenal syndrome (HSR) type I at the time of repeat paracentesis. Independent sample T-test was used to compare means of variables with normal distribution and non-parametric test (Mann U Whitney test) to compare medians of variables with nonnormal distribution. Additional analysis included changes in MELD score, serum creatinine and serum sodium levels at the time of repeat paracentesis. Results were considered significant for a $P$ value $<0.05$ and a confidence interval of $95 \%$. IBM SPSS version 19.0 was used.

\section{RESULTS}

\section{Patient Characteristics}

As shown in Figure 1, between October 2003 and June 2010, about 200 patients with cirrhosis and ascites were screened, of which, only 29 met inclusion criteria. Two patients were excluded that had loculated ascites and a large volume paracentesis $(>5 \mathrm{Lt})$ could not be performed. The remaining 27 patients were randomized, 14 to the control group (albumin), 13 to the study group (octreotide plus midodrine). Two patients (one control, one study group) were found to have spontaneous bacterial peritonitis at index LVP, had to be hospitalized and never received study medication. These two patients were withdrawn from the study. The remaining 25 patients ( 13 control, 12 study group) were included in our 
analysis; 13 from the West Haven VA, 8 from Yale-New Haven Hospital and 4 from McGuire VA. As shown in table 1, despite randomization, patients in the albumin group were younger and appeared to have more advanced liver disease as evidenced by higher Child-Pugh score, MELD score and total bilirubin. Median use of vasoconstrictors in the study group was 8 days.

\section{Time to recurrence of ascites}

Time to recurrence of ascites was not different between the two groups, with a median time to recurrence of ascites of 10 days in the control (albumin) group vs. 8 days in the study (vasoconstrictor) group $(\mathrm{p}=0.318)($ Table 2$)$. The probability of developing recurrent ascites is shown in figure 2 .

Development of PCD, HRS and assessment of hyperdynamic circulatory state Development of post-paracentesis circulatory dysfunction (PCD) defined as increase in plasma renin activity $>50 \%$ at day 6 was not significantly different between groups; $18 \%$ in the albumin group vs. $25 \%$ in the vasoconstrictor group $(\mathrm{p}=0.574)$. Change in PRA for individual patients from day 0 to day 6 in each group is shown in figures 3A and 3B. Decrease in mean arterial pressure and increase in heart rate at day 6 were also similar in both groups. None of the patients in either group developed HRS type I at day 6 or at the time of repeat paracentesis. Effect on renal function, serum sodium and MELD score

Additional analyses included comparison of serum creatinine, serum sodium and MELD score at the time of repeat paracentesis and their respective changes from baseline (Table 3). Serum creatinine at the time of repeat paracentesis was significantly higher in the vasoconstrictor (study) group compared to the albumin (control) group (1.2 vs. 0.9, $\mathrm{p}=0.051$ ), however the absolute change in serum creatinine from baseline to recurrence of ascites was not statistically significant (Table 3 ). Remarkably patients in the control group had a significant improvement in MELD score (2-point decrease) compared to patients in the study group in whom MELD score increased by a median of 0.5 points $(\mathrm{p}=0.033)$.

\section{Long Term Follow-Up}

After a median follow-up of 10 months after randomization (range 1-37), of the 25 patients randomized, , 9 patients had died (4 in the albumin group and 5 in the vasoconstrictor group), 9 patients were alive and requiring frequent paracenteses ( 5 in the albumin and 4 in vasoconstrictor group), 4 patients received orthotopic liver transplantation (two in each group), 2 patients were lost to follow-up (one in each group) and, remarkably, one patient in the albumin group has not required repeat paracentesis even one year after the index LVP (and randomization). The etiology of cirrhosis in this patient was alcohol, and his last alcohol drink had occurred 12 months prior to randomization.

\section{Adverse Events}

In the albumin group, a total of 4 adverse events were reported in 3 patients. One patient was admitted on day 3 with upper GI bleeding secondary to portal hypertensive gastropathy that resolved within 2 days; 1 patient was admitted with hepatic encephalopathy with no identified precipitant that resolved with standard therapy; 1 patient had scrotal edema and headache both of which responded to outpatient treatment. In the vasoconstrictor group, a total of 5 adverse events were reported in 4 patients. One patient had scrotal edema and diarrhea; 1 patient had diarrhea; 1 patient had pruritus and 1 patient was admitted with prerenal azotemia 1 week after completion of study. The etiology was thought to be poor oral intake and having restarted diuretics after day 6 of study. His kidney function returned to baseline after holding diuretics and volume expansion with IV albumin. 


\section{DISCUSSION}

Our pilot double-blind, placebo-controlled study was aimed to investigate the effect of a combination of vasoconstrictors, octreotide plus midodrine, compared to single use of albumin after LVP in patients with refractory ascites. Our hypothesis was that, since vasodilatation (splanchnic and systemic) plays an important role in the pathogenesis of ascites and PCD, the use of vasoconstrictors would be associated with a lower rate of PCD and thus to a longer time to recurrence of ascites compared to standard of care (serial LVPs associated with intravenous albumin at the time of paracentesis). We maximized the possibility of a positive effect by extending the effect of vasoconstrictors through the use of long-acting octreotide (with an effect that extends over 30 days) and continuing the administration of oral midodrine until recurrence of ascites. We found no significant differences in the rate of PCD between study groups and, disappointingly, we also found no differences in the time to recurrence of ascites between study groups. The short time to recurrence of ascites for both groups ( 8 and 10 days) indicates that patients included in the study were retaining sodium avidly and had true refractory ascites.

To our knowledge this is the first study investigating the effect of vasoconstrictors vs. albumin on time to recurrence of ascites after LVP. Previous randomized studies (not blinded or placebo-controlled) had compared the rate of PCD in patients treated with vasoconstrictors, compared to albumin. All these studies also included a small number of patients. Three studies used terlipressin as the vasoconstrictor, including the initial study by Moreau et al [6]. In this study and in the one by Singh et al [7], terlipressin was used intravenously at a dose of $3 \mathrm{mg} 0,8$ and 16 hours after LVP and the rate of PCD was no different in the terlipressin group (27\% in the Moreau study and $10 \%$ in the Singh study) compared to the albumin group ( $23 \%$ and $10 \%$, respectively). The third study by Lata et al. used terlipressin during 48 hours ( $1 \mathrm{mg}$ i.v. every 4 hours) and showed that terlipressin may be associated with a larger decrease in renin activity and aldosterone after LVP [8]. Terlipressin is not available in the United States and the safety of its long-term use has not been clearly established.

Therefore, we chose to use the combination of octreotide and midodrine that has been shown to be beneficial in hepatorenal syndrome [14], in which vasodilatation is also the predominant pathogenic mechanism. Two studies have investigated midodrine (without octreotide) compared to albumin in the prevention of PCD. In one of them [15], in which midodrine was administered for 3 days after LVP, the rate of PCD was very low and was not different between groups (10\% albumin, $0 \%$ midodrine), however in the other study [13], that used midodrine ( $12.5 \mathrm{mg}$ every 8 hours) for 2 days after LVP, the rate of PCD was higher in the midodrine group (6/11 or $61 \%$ ) compared to the albumin group (4/13 or $31 \%)$. An uncontrolled study by Tandon et al [16] analyzed 8 patients treated with octreotide, midodrine and albumin during one month, and found a trend towards a lower volume of ascites removed by LVP while on therapy and a reduction in PRA and aldosterone, without changes in renal function. However, there was a transient worsening in the MELD score that reversed after discontinuation of therapy. In our study, and even though not statistically different, the outcomes in the albumin group were somewhat better, with a time to recurrence of ascites of 10 days (compared to 8 days in the vasoconstrictor group) and a rate of PCD of $18 \%$ compared to $25 \%$ in the vasoconstrictor group. These differences take a greater significance if one considers that our study groups were somewhat unequal at baseline with patients in the control (albumin) group being somewhat sicker than the study (vasoconstrictor) group, as reflected by a higher Child score. Of more concern is the fact that, at the time of recurrence of ascites, serum creatinine levels were significantly higher in patients that received octreotide plus midodrine compared to those that received standard of 
care (albumin) and that, while MELD score decreased in patients in the control group, it increased slightly in the vasoconstrictor group.

These findings are consistent with prior studies described above that have reported a higher rate of PCD with midodrine and a worsening MELD with the combination octreotide/ midodrine $[13 ; 16]$. Therefore, it would appear that vasoconstrictors, specifically the combination of octreotide plus midodrine, demonstrate no advantage over albumin after LVP on the time to recurrent ascites and may have a deleterious effect on renal function. Notably, a recent meta-analysis that evaluated randomized trials comparing albumin with alternative therapies (synthetic plasma expanders, hypertonic saline or vasoconstrictors) after LVP, concluded that albumin significantly reduces morbidity and mortality among patients with tense ascites undergoing LVP, as compared with alternative treatments [17]. Given the small number of patients enrolled in trials comparing albumin and vasoconstrictors, the results of the meta-analysis were not as robust when only trials of albumin vs. vasoconstrictors were analyzed although a trend favoring albumin was still observed [17].

Our study is limited because of the small sample size and the fact that patients received a fixed dose of midodrine/octreotide, not titrated to increases in mean arterial pressure, and that, although adherence to octreotide was not an issue (given the administration by research personnel of long-acting octreotide) adherence to midodrine could not be evaluated.

However, in the four patients in the vasoconstrictor group in whom MAP had increased at day 6 , one patient still developed PCD without any significant changes in serum creatinine or MELD score. In summary, in a double-blind, placebo-controlled proof-of-concept study, long-term administration of a combination of orally administered midodrine and intramuscular long-acting octreotide was not superior to albumin in delaying the recurrence of ascites after LVP or in preventing PCD. In fact, outcomes appear to be worse in patients who received the combination vasoconstrictor therapy. Given these results, the performance of larger trials would not be warranted using octreotide/midodrine and similar proof-ofconcept studies using a more potent vasoconstrictor, such as terlipressin, should be undertaken.

\section{Acknowledgments}

Grant support: VA Merit Review Grant and NIH P-30DK 034989

\section{Abbreviations}

$\begin{array}{ll}\text { LVP } & \text { Large volume paracentesis } \\ \text { PCD } & \text { Post-paracentesis circulatory dysfunction } \\ \text { PRA } & \text { Plasma renin activity } \\ \text { IM } & \text { Intramuscular } \\ \text { MAP } & \text { Mean arterial pressure } \\ \text { BUN } & \text { Blood urea nitrogen } \\ \text { HRS } & \text { Hepatorenal syndrome } \\ \text { MELD } & \text { Model for end-stage liver disease }\end{array}$




\section{References}

1. Arroyo V, Gines P, Gerbes AL, et al. Definition and diagnostic criteria of refractory ascites and hepatorenal syndrome in cirrhosis. Hepatology. 1996; 23:164-176. [PubMed: 8550036]

2. EASL clinical practice guidelines on the management of ascites, spontaneous bacterial peritonitis, and hepatorenal syndrome in cirrhosis. J Hepatol. 2010; 53:397-417. [PubMed: 20633946]

3. Gines A, Fernandez-Esparrach G, Monescillo A, et al. Randomized trial comparing albumin, dextran-70 and polygeline in cirrhotic patients with ascites treated by paracentesis. Gastroenterology. 1996; 111:1002-1010. [PubMed: 8831595]

4. Ruiz del Arbol L, Monescillo A, Jimenez W, et al. Paracentesis-induced circulatory dysfunction: mechanism and effect on hepatic hemodynamics in cirrhosis. Gastroenterology. 1997; 113:579-586. [PubMed: 9247479]

5. Sola-Vera J, Minana J, Ricart E, et al. Randomized trial comparing albumin and saline in the prevention of paracentesis-induced circulatory dysfunction in cirrhotic patients with ascites. Hepatology. 2003; 37:1147-1153. [PubMed: 12717396]

6. Moreau R, Asselah T, Condat B, et al. Comparison of the effect of terlipressin and albumin on arterial blood volume in patients with cirrhosis and tense ascites treated by paracentesis: a randomized pilot study. Gut. 2002; 50:90-94. [PubMed: 11772973]

7. Singh V, Kumar R, Nain CK, et al. Terlipressin versus albumin in paracentesis-induced circulatory dysfunction in cirrhosis: a randomized study. J Gastroenterol Hepatol. 2006; 21:303-307. [PubMed: 16460491]

8. Lata J, Marecek Z, Fejfar T, et al. The efficacy of terlipressin in comparison with albumin in the prevention of circulatory changes after the paracentesis of tense ascites--a randomized multicentric study. Hepatogastroenterology. 2007; 54:1930-1933. [PubMed: 18251131]

9. Moore KP, Wong F, Gines P, et al. The management of ascites in cirrhosis: report on the consensus conference of the International Ascites Club. Hepatology. 2003; 38:258-266. [PubMed: 12830009]

10. Thomsen TW, Shaffer RW, White B, et al. Videos in clinical medicine. Paracentesis. N.Engl.J Med. 2006; 355:e21. [PubMed: 17093242]

11. Salerno F, Gerbes A, Gines P, et al. Diagnosis, prevention and treatment of the hepatorenal syndrome in cirrhosis. A consensus workshop of the international ascites club. Gut. 2007; 56:1310-1318. [PubMed: 17389705]

12. Gines P, Uriz J, Calahorra B, et al. Transjugular intrahepatic portosystemic shunting versus repeated paracentesis plus intravenous albumin for refractory ascites in cirrhosis: A multicenter randomized comparative study. Gastroenterology. 2002; 123:1839-1847. [PubMed: 12454841]

13. Appenrodt B, Wolf A, Grunhage F, et al. Prevention of paracentesis-induced circulatory dysfunction: midodrine vs albumin. A randomized pilot study. Liver Int. 2008; 28:1019-1025. [PubMed: 18410283]

14. Angeli P, Volpin R, Gerunda G, et al. Reversal of type 1 hepatorenal syndrome with the administration of midodrine and octreotide. Hepatology. 1999; 29:1690-1697. [PubMed: 10347109]

15. Singh V, Dheerendra PC, Singh B, et al. Midodrine versus albumin in the prevention of paracentesis-induced circulatory dysfunction in cirrhotics: a randomized pilot study. Am J Gastroenterol. 2008; 103:1399-1405. [PubMed: 18547224]

16. Tandon P, Tsuyuki RT, Mitchell L, et al. The effect of 1 month of therapy with midodrine, octreotide-LAR and albumin in refractory ascites: a pilot study. Liver Int. 2009; 29:169-174. [PubMed: 18492024]

17. Bernardi M, Caraceni P, Navickis RJ, Wilkes MM. Albumin infusion in patients undergoing largevolume paracentesis: A meta-analysis of randomized trials. Hepatology. 2012; 55:1172-1181. [PubMed: 22095893] 


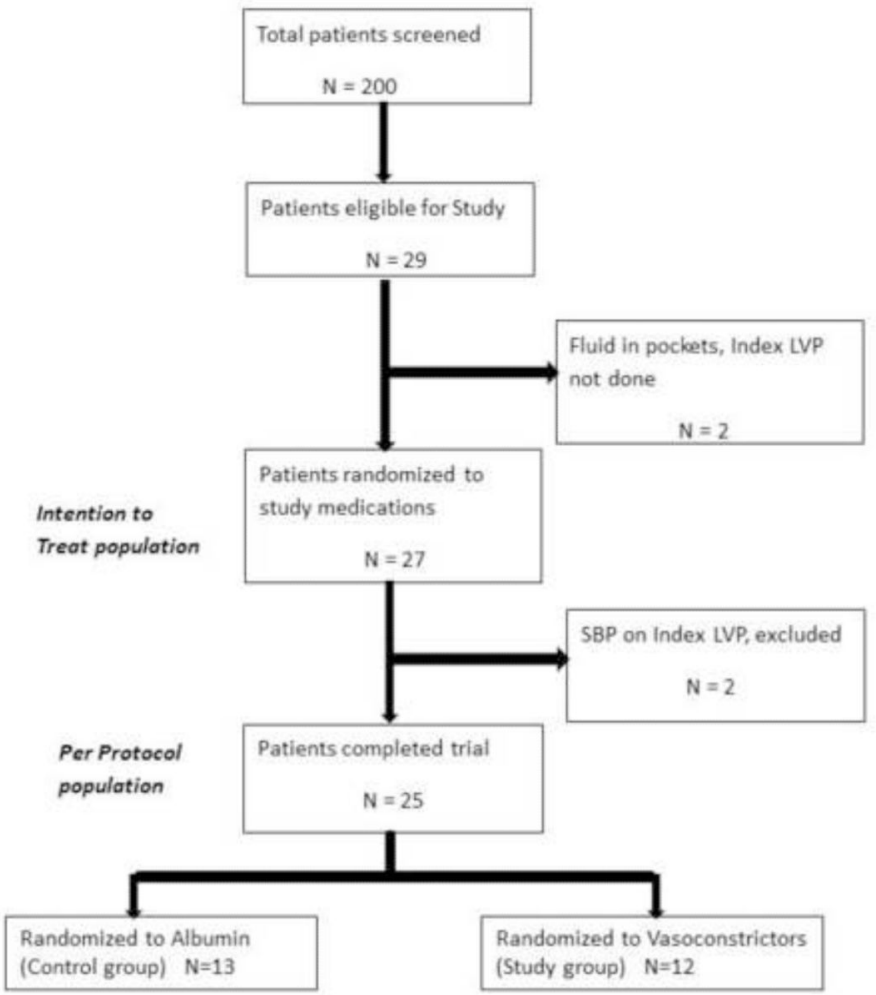

Figure 1.

Flowchart of patients considered and entered into the study 


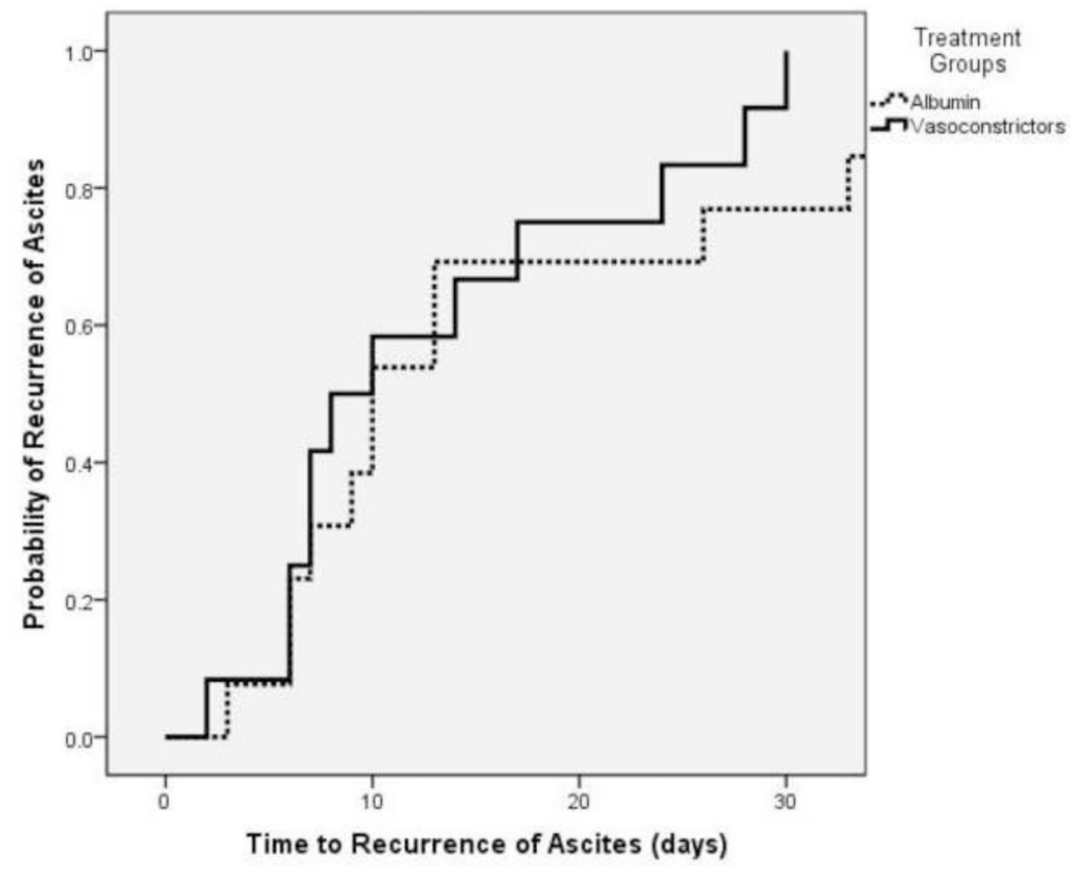

Figure 2.

Probability of developing recurrent ascites in the albumin (control) group compared to the vasoconstrictor (study) group. There were no differences in recurrence of ascites with a median time to recurrence of 10 days in the albumin group and 8 days in the vasoconstrictor group $(\mathrm{p}=0.318)$ 

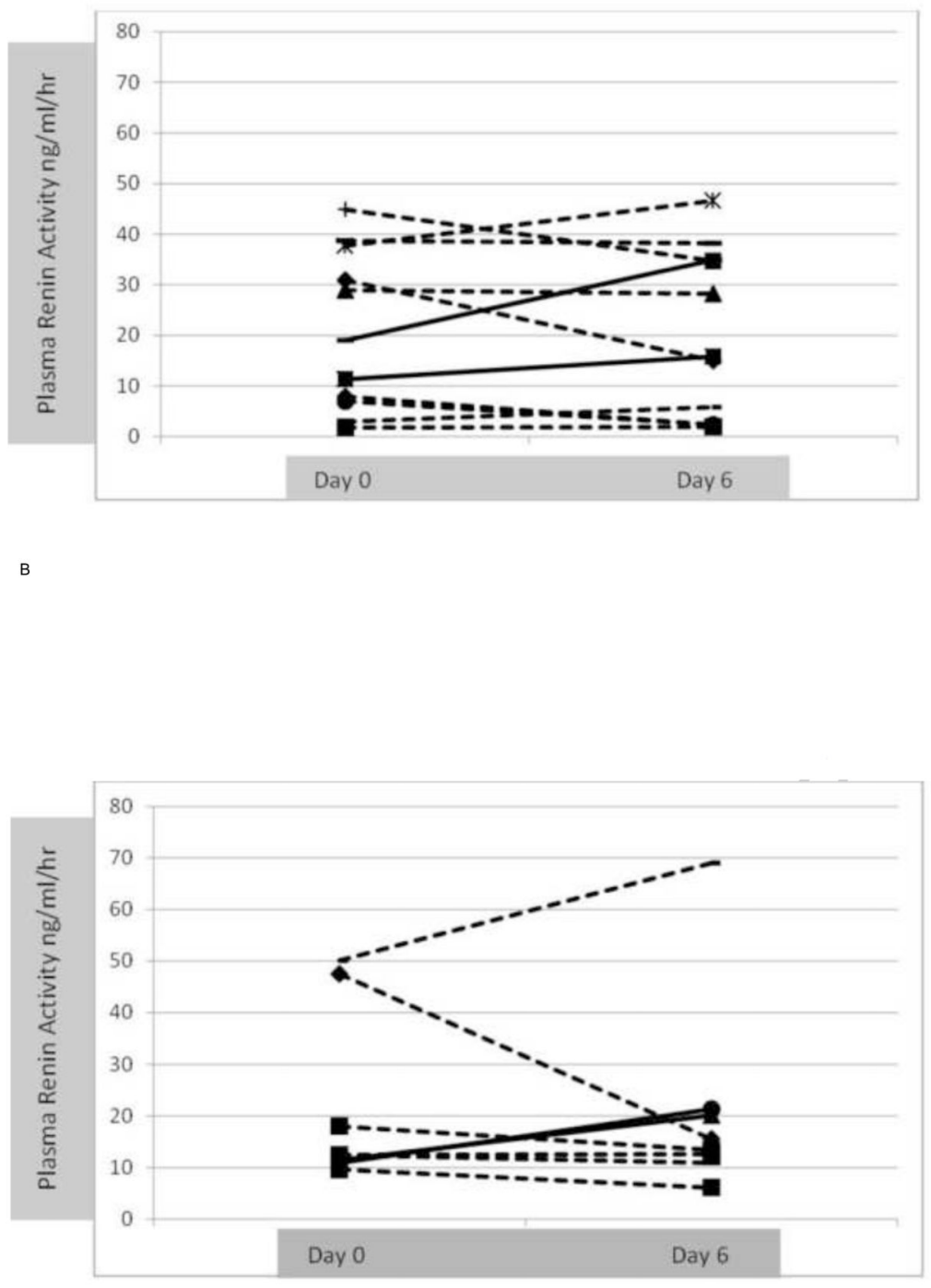

Figure 3.

A. Changes in individual plasma renin activity at randomization (day 1) and at day 6 in the albumin (control group). 2/11 (18\%) patients (continuous line) developed post-paracentesis circulatory dysfunction

B. Changes in individual plasma renin activity at randomization (day 1) and at day 6 in the vasoconstrictor (study group). 2/8 (25\%) patients (continuous line) developed postparacentesis circulatory dysfunction. 
Table 1

Baseline characteristics of patients included in the study

\begin{tabular}{|c|c|c|c|}
\hline & $\underset{\text { (control group) }}{\text { Albumin }}$ & $\begin{array}{l}\text { Vasoconstrictors } \\
\text { (study group) }\end{array}$ & $\mathbf{P}$ \\
\hline $\mathbf{N}$ & 13 & 12 & \\
\hline Age (years) & $55(51-61)$ & $60(51-65)$ & 0.398 \\
\hline Gender (Male) & $10(77 \%)$ & $12(100 \%)$ & 0.124 \\
\hline $\begin{array}{l}\text { Race } \\
\text { White } \\
\text { African American } \\
\text { Hispanic } \\
\text { Middle eastern }\end{array}$ & $\begin{array}{c}9(69 \%) \\
3(23 \%) \\
0 \\
1(8 \%)\end{array}$ & $\begin{array}{l}9(75 \%) \\
2(17 \%) \\
1(8 \%) \\
0\end{array}$ & 0.539 \\
\hline $\begin{array}{l}\text { Etiology of cirrhosis } \\
\text { Alcohol } \\
\text { HCV } \\
\text { Alcohol + HCV } \\
\text { Other }{ }^{*}\end{array}$ & $\begin{array}{l}5(38 \%) \\
1(8 \%) \\
4(31 \%) \\
3(23 \%)\end{array}$ & $\begin{array}{l}8(66 \%) \\
1(8 \%) \\
1(8 \%) \\
2(17 \%)\end{array}$ & 0.280 \\
\hline $\begin{array}{l}\text { Last alcohol drink } \\
\text { Between } 1 \text { week and } 1 \text { month } \\
\text { Between } 1 \text { to } 3 \text { months } \\
\text { Between } 3 \text { to } 6 \text { months } \\
\text { Between } 6 \text { to } 12 \text { months } \\
\text { More than } 12 \text { months ago }\end{array}$ & $\begin{array}{l}1 / 9^{* *}(11 \%) \\
2 / 9(22 \%) \\
1 / 9(11 \%) \\
3 / 9(34 \%) \\
2 / 9(22 \%)\end{array}$ & $\begin{array}{l}1 / 9^{* *}(11 \%) \\
1 / 9(11 \%) \\
3 / 9(34 \%) \\
2 / 9(22 \%) \\
2 / 9(22 \%)\end{array}$ & 0.821 \\
\hline On non-selective beta-blockers & $6(46 \%)$ & $7(58 \%)$ & 0.695 \\
\hline On antibiotic prophylaxis & 0 & $3(25 \%)$ & 0.096 \\
\hline Mean arterial pressure $(\mathrm{mm} \mathrm{Hg})$ & $78(72-89)$ & $84(74-96)$ & 0.264 \\
\hline Heart rate (beats/min) & $82(63-93)$ & $78(72-89)$ & 0.913 \\
\hline Amount of ascites removed (liters) & $6.5(5-9.5)$ & $8(6-10.5)$ & 0.354 \\
\hline Ascitic fluid total protein $(\mathrm{g} / \mathrm{dl})$ & $1.1(0.9-1.41)$ & $1.2(0.9-1.5)$ & 0.705 \\
\hline Child Pugh score & $10(9-11)$ & $8(8-10)$ & 0.055 \\
\hline MELD score & $17(11-20)$ & $14(13-16)$ & 0.200 \\
\hline Hemoglobin (g/dl) & $11.1(9.5-11.9)$ & $11.3(8.9-13.2)$ & 0.605 \\
\hline Hematocrit $\%$ & $31(28-36)$ & $33(27-38)$ & 0.568 \\
\hline Platelet count $\left(1000 / \mathrm{cm}^{2}\right)$ & $122(95-213)$ & $109(75-207)$ & 0.663 \\
\hline INR & $1.5(1.3-1.6)$ & $1.4(1.3-1.5)$ & 0.304 \\
\hline Blood urea nitrogen $(\mathrm{mg} / \mathrm{d}) 1$ & $20(13-29)$ & $24(16-35)$ & 0.384 \\
\hline Creatinine $(\mathrm{mg} / \mathrm{dl})$ & $1.1(0.9-1.5)$ & $1.1(1-1.5)$ & 0.805 \\
\hline Serum sodium $(\mathrm{mmol} / \mathrm{l})$ & $134(131-135)$ & 136(131-137) & 0.566 \\
\hline Albumin $(\mathrm{g} / \mathrm{dl})$ & $2.5(1.8-3)$ & $2.8(2.2-2.3)$ & 0.327 \\
\hline Total bilirubin (mg/dl) & $3.4(1.7-4.2)$ & $1.8(1.5-3.2)$ & 0.142 \\
\hline $\operatorname{ALT}(\mathrm{U} / \mathrm{L})$ & $40(35-80)$ & $29(22-49)$ & 0.022 \\
\hline $\operatorname{AST}(\mathrm{U} / \mathrm{L})$ & $28(20-58)$ & $30(16-41)$ & 0.414 \\
\hline Urine sodium $(\mathrm{mmol} / \mathrm{L})$ & $33(21-50)$ & $23(15-29)$ & 0.474 \\
\hline Plasma renin activity (ng/ml/hr) & $19(17.4-34.5)$ & $11.8(7.9-25.1)$ & 0.828 \\
\hline Serum aldosterone (ng/dl) & $36(18-89)$ & $42(12-100)$ & 0.550 \\
\hline
\end{tabular}

Values are expressed as percentages, medians and interquartile ranges. 
2 autoimmune, 1 non-alcoholic steatohepatitis, 1 PBC, 1 cryptogenic

Only patients with alcoholic etiology of cirrhosis 


\section{Table 2}

Comparison of primary and secondary outcomes by study group

\begin{tabular}{lccc}
\hline Outcome & $\begin{array}{c}\text { Albumin } \\
\text { (control group) }\end{array}$ & $\begin{array}{c}\text { Vasoconstrictors } \\
\text { (study group) }\end{array}$ & P \\
$\mathrm{N}$ & 13 & 12 & 0.318 \\
$\begin{array}{l}\text { Time to recurrence of ascites } \\
\text { (days) }\end{array}$ & $10(7-29)$ & $8(6-22)$ & 0.574 \\
$\begin{array}{l}\text { Patients who developed PCD (\%) } \\
\text { Change in PRA at day 6 } \\
\text { (ng/mL/hr) }\end{array}$ & $2 / 11(18 \%)$ & $2 / 8(25 \%)$ & 0.741 \\
$\begin{array}{l}\text { Change in heart rate at day 6 } \\
\text { (bpm) }\end{array}$ & $\downarrow 1.3(-51-40)$ & $\uparrow 7.1(-22-67)$ & \\
$\begin{array}{l}\text { Change in MAP at day 6 (mmHg) } \\
\text { Development of HRS at Day 6 }\end{array}$ & $\downarrow 5(-7-2)$ & $\downarrow 2(-7-5)$ & 0.675 \\
\hline
\end{tabular}

Results are expressed as medians (interquartile range);

*

6-day PRA levels were available in 11 patients in the control group and 8 in the study group. PCD=post-paracentesis circulatory dysfunction; $\mathrm{PRA}=$ plasma renin activity; $\mathrm{MAP}=$ mean arterial pressure; $\mathrm{HRS}=$ hepatorenal syndrome 
Table 3

Serum creatinine, sodium and MELD score and changes in these parameters (compared to baseline) at the time of recurrence of ascites (repeat paracentesis)

\begin{tabular}{|l|c|c|c|}
\hline & Albumin (control group) & Vasoconstrictors (study group) & $\mathbf{p}$ \\
\hline $\mathrm{N}$ & $11^{*}$ & $9^{*}$ & \\
\hline Serum creatinine $-(\mathrm{mg} / \mathrm{dl})$ & $0.9(0.9-1.4)$ & $1.2(1.0-1.8)$ & 0.051 \\
\hline Change in serum creatinine $(\mathrm{mg} / \mathrm{dl})$ & $0(-0.2-0.2)$ & $\uparrow 0.1(-0.4-0.1)$ & 0.064 \\
\hline Serum sodium $(\mathrm{mmol} / \mathrm{L})$ & $133(131-135)$ & $134(129-136)$ & 0.974 \\
\hline Change in serum sodium $(\mathrm{mmol} / \mathrm{l})$ & $\downarrow 1(-3-0)$ & $\downarrow 1(-3-1)$ & 0.868 \\
\hline MELD score & $14(10-16)$ & $15(12-18)$ & 0.203 \\
\hline Change in MELD score & $\downarrow 2(-3-1)$ & $\uparrow 0.5(-1-3.5)$ & 0.033 \\
\hline
\end{tabular}

not all patients randomized had repeat tests at the time of repeat paracentesis 